VOL. 9 (1973), 49-54.

\title{
Conditions for a plane projective metric to be a norm
}

\section{B.B. Phadke}

\begin{abstract}
Let $R$ be a metrization with distance $x y$ of an open convex set $D$ in the 2-dimensional real affine plane such that $x y+y z=x z$ whenever $x, y, z$ lie on an affine line with $y$ between $x$ and $z$ and such that all the balls $p x \leq \rho$ are compact. The study of such metrics, called open plane projective metrics falls under the topic of Hilbert's Problem IV of his famous mathematical problems. In this paper it is proved that if in $R$ the sets of points equidistant from lines lie again on lines then $D$ must be the entire affine plane and the distance must in fact be a norm. The paper contributes to and gives extensions of similar results proved earlier. The novel features of the present result are that in the space collinearity of points $x, y, z$ is taken only as a sufficient condition for the equality $x y+y z=x z$. Consequently the solution encompasses all normed linear planes, that is, norms whose unit circles are not necessarily strictly convex are also admitted.
\end{abstract}

\section{The background}

The idea of using a flatness condition on the equidistant loci is due to Funk [4]. However, he considered only the case when $D$ is either the entire affine plane or the interior of a strictly convex closed curve. Also his metric was finslerian. He showed that we obtain a minkowskian (normed linear) geometry or his "Geometrie der spezifischen

Received 27 February 1973. 
Massbestimmung", see [3, p. 232], when equidistant loci are lines locally, that is an affine segment $S$ in the space has a convex neighbourhood $u$ such that the points $x$ in $U$ at a constant distance from the line containing $S$ lie on lines. Phadke [5] considered the problem in $n$ dimensions in the context of a straight desarguesian G-space (that is, the space $R$ as above except that $x y+y z=x z$ if and only if $x, y, z$ lie on an affine segment in that order). This formulation excluded all normed linear spaces whose unit balls are not strictly convex because in a straight $G$-space spheres are strictly convex whenever they are convex. Recently Busemann [2] generalized his $G$-spaces to include spaces in which segments are not unique even locally. These spaces are called "chord spaces". In a straight chord space convexity of balls does not always imply strict convexity. The space $R$ of the present paper is a straight desarguesian chord space. Thus we show that the property of flatness of equidistant loci can be used to characterize the class of all normed linear planes among the wider class of all straight desarguesian chord planes.

\section{Statement of the theorem}

Let $R$ be a space as defined at the beginning of the paper. To formulate our result, for a set $M$ and a point $p$ define $M p=\inf \{m p \mid m \in M\}$ and for $\alpha \geq 0$ the equidistant locus $E(M, \alpha)=\{x \mid M x=\alpha\}$. Denoting the two sides of a line $L$ by $\sigma^{ \pm}(L)$, we define $E^{ \pm}(L, \alpha)=E(L, \alpha) \cap \sigma^{ \pm}(L)$. We prove the following.

THEOREM. The space $R$ is a normed linear space if and only if the equidistants of affine lines are again affine lines.

A word about notation. Whenever $M p=q p$ we say that $q$ is a foot of $p$ on $M$. The notation $(p q r)$ means that $p, q, r$ satisfy $p q+q r=p r$. When $p, q, r$ are points of an affine segment with $q$ lying between $p$ and $r$ we will write $[p q r]$. The symbol $F(p, L)$ stands for the set of feet of $p$ on $L, Q(a, b)$ denotes the affine segment joining $a$ and $b$.

\section{Convexity of circles and the perpendicularity}

To prove the theorem stated above the major step is to show that $D$ must be the entire affine plane. We first show that the circles must be 
convex, that is, whenever $u$ and $v$ lie in the disc $p x \leq \rho$ the affine segment joining $u$ and $v$ also lies in that disc. Note first that $L_{1}=E\left(L_{2}, \alpha\right)$ implies $L_{2}=E\left(L_{1}, \alpha\right)$ because choosing $x \in L_{1}, y \in L_{2}$ with $x y=\alpha$ we draw $L_{3}$ through $y$ such that $L_{3}=E\left(L_{1}, \alpha\right)$. If $L_{3}$ did not coincide with $L_{2}$ then $L_{3}$ would contain points $z$ on the side of $L_{2}$ not containing $L_{1}$. All these points are at a distance greater than $\alpha$ which is a contradiction to $z \in E\left(L_{1}, \alpha\right)$.

We use this fact to prove that the set of feet of a point on a line is a connected set. Let $x, y \in F(p, L), x p=y p=\alpha$. If $L^{\prime}=E(L, \alpha)$ with $p \in L$ and [xmy] then $m$ has a foot $n$ on $L^{\prime} . Q(m, n)$ meets either $Q(p, y)$ or $Q(p, x)$, say, $Q(p, y)$ in $z$. If $z_{m}>z_{y}$ we would have $n y \leq n l+l y<n l+l m=n m=\alpha$. But $n y<\alpha$ contradicts the symmetry of "being equidistant" which was proved above. Hence $l_{m} \leq l_{y}$. Consequently $\alpha \leq p m \leq p l+l m \leq p l+l y=p y=\alpha$. Thus $m$ is also a foot of $p$ on $L$. This proves that $F(p, L)$ is a connected set for all $p$ and all $L$. A result of Busemann [2, p. 115] now implies that all the circles $p x \leq \rho$ are convex.

A line $H$ is said to be perpendicular to the line $L$ if $H$ intersects $L$ in a point $f$ and each point of $H$ has $f$ as its foot on $L$. Busemann's results [2, p. 115] again imply that when all the circles are convex each $p \notin L$ is the origin of perpendiculars whose union intersects $L$ in a point or an affine segment. We note that although perpendiculars exist, they are not unique (which was the case in Phadke [5]).

\section{Classification of the domains $D$}

Although the perpendicuiars are not unique the following still holds. Given two equidistants $L_{1}$ and $L_{2}$ and a point $x$ between them there exists a common perpendicular to $L_{i}$ through $x$. In fact let $H$ be a perpendicular through $x$ to $L_{1}$. Let $H$ meet $L_{1}$ in $p$ and suppose $L_{2}=E\left(L_{1}, \alpha\right)$. Take $q \in H$ with $q p=\alpha$. Since $H$ is perpendicular to $L_{1}, p$ is a foot of $q$ on $L_{1}$. Hence $q$ is at a distance $\alpha$ from 
$L_{1}$. Therefore $q$ lies on $L_{2}$. Now by the symmetry of the relation of "being equidistant" $q$ is also a foot of $p$ on $L_{2}$. Hence the line joining $q$ to $p$, that is $H$, is perpendicutar to $L_{2}$ also. (See Busemann [2, p. 115].)

In virtue of the above observation no extreme point of the affine closure $\bar{D}$ of $D$ can lie between two equidistants. Hence we can prove in the same manner as in Phadke [5, p. 315] that $D$ must either be a triangle or a strip enclosed by two affine parallel lines or the entire affine plane.

\section{5. $D$ is the entire affine plane}

In this section we prove that our geometry cannot be defined inside a triangle or a strip. The proof of the impossibility of the strip as given in Phadke [5, p. 315, 316] does not need any change except one writes " $a$ perpendicular" in place of "the perpendicular". Hence we consider only the case of a triangle. Even in this case we can prove as in [5, p. 315] that all equidistants of a line through a vertex must again pass through the same vertex and that if a line does not pass through a vertex then all the perpendiculars to that line pass through a vertex. However, the rest of the proof given there cannot be carried through without modifications because that proof used the continuous change of feet and perpendiculars which is not available to us now because the feet and the perpendiculars are no longer unique.

Nevertheless we prove that lines through one vertex are perpendicular to lines through another vertex. Let $a, b, c$ be the vertices of the triangle $D$. Let $L$ be a line passing through the vertex $a$ and let $b \in \sigma^{-}(L)$ and $c \in \sigma^{+}(L)$. Let $x \in \sigma^{+}(L)$ have $p \in L$ as its foot. If [cxp] does not hold let the line joining $c$ and $x$ meet $L$ in $q$. We prove $x q=x p$. For if $x p<x q$ we can find a point $u$ with [xuq] such that $x p<x u$ and the line $L^{\prime}$ joining $u$ and $p$ does not pass through a vertex. But then the perpendicular to $L^{\prime}$ through $x$ must pass through $c$ which is a contradiction to $x u>x p$. Thus lines passing through a vertex are always perpendiculars. This implies that the line joining $c$ and $p$ is perperdicular to all other lines through $p$. This 
is clearly impossible because otherwise a circle through $p$ would shrink to a segment on $Q(c, p)$. Thus the geometry cannot be defined inside a triangle. As noted at the beginning of this section this implies that the geometry can only be defined in the entire affine plane.

\section{The metric is a norm}

Since the geometry is defined in the entire affine plane, the equidistant of a line is its affine parallel. We will show that every metric circle $p x=\rho$ possesses parallel supporting lines at the endpoints of every chord (diameter) through the centre. In fact if $L$ is a supporting line to the circle to an endpoint $a$ of a diameter $H$ then $H$ is a perpendicular to $L$. Consequently the equidistant to $L$ through the other end of $H$ is also a supporting line of the circle. Since equidistant lines are affine parallels we have proved our assertion. By a result of Busemann [1, p. 91] we know that a closed convex curve $C$ has a point $z$ inside it as its affine centre if $C$ possesses parallel supporting lines at the endpoints of every chord through $z$. Hence in our geometry the metric centre $p$ of a circle $p x=\rho$ is also its affine centre. Therefore for any segment the metric midpoint is the same as its affine midpoint. It is well known, see Busemann [1, p. 94], that this implies that the metric is a norm. This completes the proof of our theore theorem.

\section{References}

[1] Herbert Busemann, The geometry of geodesics (Academic Press, New York, 1955).

[2] Herbert Busemann, "Spaces with distinguished shortest joins", A spectrum of mathematics, 108-120 (essays presented to H.G. Forder; edited by J.C. Butcher; University Press, Auckland, New Zealand; Oxford University Press, Oxford; 1971).

[3] Paul Funk, "Über Geometrien, bei denen die Geraden die Kürzesten sind", Math. Ann. 101 (1929), 226-237.

[4] P. Funk, "Über Geometrien, bei denen die Geraden die kürzesten Linien sind und die Aquidistanten zu einer Geraden wieder Gerade sind", Monatshefte Math: Phys. 37 (1930), 153-158. 
[5] B.B. Phadke, "Equidistant loci and the Minkowskian geometries", Canad. J. Math. 24 (1972), 312-327.

School of Mathematical Sciences,

Flinders University,

Bedford Park,

South Australia. 